\title{
Ingestão de energia e de nutrientes e baixo peso ao nascer: estudo de coorte com gestantes adolescentes ${ }^{1}$
}

\author{
Energy and nutrient intakes and low \\ birth weight: Cohort study with \\ pregnant adolescents
}

\author{
Aline Bull Ferreira CAMPOS² \\ Rosangela Alves PEREIRA ${ }^{3}$ \\ Juliana QUEIROZ² \\ Cláudia SAUNDERS ${ }^{4}$
}

RE S U M O

\section{Objetivo}

Avaliar a ingestão de energia, de macro e de micronutrientes por adolescentes no primeiro e no segundo trimestres de gestação e estimar sua associação com o peso do concepto ao nascer.

\section{Métodos}

Estudo longitudinal, incluindo 139 gestantes adolescentes atendidas em serviço de pré-natal de maternidade pública, acompanhadas desde o primeiro trimestre gestacional até o puerpério imediato. Aplicou-se um questionário de frequência de consumo alimentar no primeiro e no segundo trimestres de gestação. O peso e a idade gestacional ao nascer dos recém-nascidos foram coletados dos prontuários hospitalares. Foram estimados médias e intervalo de confiança de $95 \%$. O método dos resíduos foi utilizado para ajustar o consumo de nutrientes pelo consumo energético total. Modelos de regressão linear múltipla foram desenvolvidos para identificar os fatores associados ao peso ao nascer.

\section{Resultados}

O consumo médio de proteínas ( $p=0,02)$, lipídeos $(p=0,02)$, ácidos graxos saturados $(p=0,02)$ e monoinsaturados $(p=0,05)$, colesterol $(p=0,01)$, cálcio $(p \leq 0,01)$, potássio $(p=0,01)$ e fósforo $(p \leq 0,01)$ foi mais elevado entre

\footnotetext{
1 Artigo elaborado a partir da dissertação de ABF CAMPOS, intitulada "Associação entre o consumo de energia e nutrientes e peso ao nascer em gestantes adolescentes”. Universidade Federal do Rio de Janeiro; 2011.

2 Universidade Federal do Rio de Janeiro, Instituto de Nutrição Josué de Castro, Programa de Pós-Graduação em Nutrição. Av. Carlos Chagas Filho, 373, Bloco J, $2^{\circ}$ andar, Sala 26, Ilha do Fundão, 21941-902, Rio de Janeiro, RJ, Brasil. Correspondência para/Correspondence to: ABF CAMPOS. E-mail: <alinebull@yahoo.com.br>.

3 Universidade Federal do Rio de Janeiro, Instituto de Nutrição Josué de Castro, Departamento de Nutrição Social e Aplicada. Rio de Janeiro, RJ, Brasil.

${ }^{4}$ Universidade Federal do Rio de Janeiro, Instituto de Nutrição Josué de Castro, Departamento de Nutrição e Dietética. Rio de Janeiro, RJ, Brasil.
} 
552 | ABF CAMPOS et al.

gestantes que tiveram filhos com peso ao nascer acima de $2500 \mathrm{~g}$. Idade gestacional ao parto $(\beta=105,8 ; p<0,01)$, número de consultas de pré-natal $(\beta=34,1 ; p=0,04)$, consumo de ácidos graxos poli-insaturados $(\beta=7,5 ; p=0,04)$ e de ômega-3 $(\beta=74,3 ; p=0,05)$ e de colesterol $(\beta=0,4 ; p=0,04)$ foram variáveis preditoras do peso ao nascer em modelos de regressão linear múltipla.

\section{Conclusão}

O perfil lipídico da dieta da mãe foi o principal preditor do peso do concepto ao nascer, e os resultados sugerem que o acompanhamento nutricional deva ser incluído na rotina dos serviços de atenção pré-natal.

Termos de indexação: Consumo de alimentos. Cuidado pré-natal. Gravidez na adolescência. Peso ao nascer.

\section{A B S T R A C T}

\section{Objective}

This study assessed energy, macronutrient, and micronutrient intakes of adolescents in the first and second trimesters of pregnancy and investigated their association with the infant's birth weight.

\section{Methods}

This longitudinal study included 139 pregnant adolescents seen at the prenatal care clinic of a public maternity hospital. They were followed from the first trimester of pregnancy until hospital discharge after delivery. The participants answered a food frequency questionnaire in the first and second trimesters of pregnancy. Birth weight and gestational age were collected from the mothers' hospital records. The means and 95\% confidence intervals were calculated. The residual method was used for adjusting nutrient intake according to total energy intake. The factors associated with birth weight were identified by multiple linear regression models.

\section{Results}

The mothers of infants with birth weight higher than 2500 grams had higher mean intakes of protein $(p=0.02)$, lipids $(p=0.02)$, saturated fatty acids $(p=0.02)$ monounsaturated fatty acids $(p=0.05)$, cholesterol $(p=0.01)$, calcium ( $p \leq 0.01)$, potassium $(p=0.01)$, and phosphorus $(p \leq 0.01)$. According to the multiple linear regression models, gestational age at delivery $(\beta=105.8 ; \beta<0.01)$, number of prenatal care visits $(\beta=34.1 ; p=0.04)$, intake of polyunsaturated fatty acids ( $\beta=7.5 ; \quad p=0.04)$, intake of omega-3 fatty acids $(\beta=74.3 ; p=0.05)$, and cholesterol intake $(\beta=0.4 ; p=0.04$ ) were predictors of birth weight.

\section{Conclusion}

The mother's dietary lipid profile was the main predictor of the infant's birth weight and the results suggest that nutritional follow-up should be included in the prenatal care routine.

Indexing terms: Food consumption. Prenatal care. Pregnancy in adolescence. Birth weight.

\section{N T R O D U Ç Ã O}

A ocorrência de gestação na adolescência cresceu de forma constante nos últimos anos, o que despertou o interesse científico pelas consequências clínicas, nutricionais e psicológicas da gravidez e do parto em fase de pleno processo de desenvolvimento físico e psicológico ${ }^{1}$. Devido à imaturidade fisiológica para suportar o estresse da gestação, a maternidade em idade precoce representa risco elevado de resultado obstétrico desfavorável, principalmente de deficit no crescimento fetal, parto prematuro e baixo peso ao nascer ${ }^{1}$.
As necessidades nutricionais são aumentadas durante a gestação. Esse aumento é ainda maior nas adolescentes, pois além do aporte extraordinário de energia e de nutrientes necessário ao desenvolvimento fetal ${ }^{2}$, elas devem receber alimentação em quantidade e qualidade suficientes para o crescimento físico e desenvolvimento fisiológico típicos da adolescência ${ }^{3}$. A dieta materna durante a gravidez pode influenciar a suscetibilidade individual às complicações perinatais. Estudos mais recentes têm mostrado que a nutrição durante o desenvolvimento fetal se associa ao desenvolvimento de enfermidades na vida adulta ${ }^{4}$. 
No Brasil, o consumo alimentar de adolescentes grávidas tem sido considerado inadequado em relação à energia e a diversos nutrientes, como ferro, folato e cálcio, que podem, eventualmente, favorecer o desenvolvimento de distúrbios nutricionais na mãe ou no concepto ${ }^{3,5}$. São escassos, entretanto, os estudos que avaliam a associação entre consumo alimentar durante a gestação e desfechos perinatais, como, por exemplo, o peso ao nascer, que é considerado um indicador sensível do estado nutricional intrauterino e pós-natal e um importante preditor de vulnerabilidade a fatores ambientais e sociais de risco para o aumento da morbidade e da mortalidade durante o primeiro ano de vida ${ }^{6}$.

O presente trabalho teve como objetivo descrever e avaliar a ingestão de energia, de macro e de micronutrientes no primeiro e no segundo trimestres de gestação e sua associação com o resultado perinatal em adolescentes atendidas em serviço público de atenção pré-natal no município do Rio de Janeiro.

\section{MÉTODOS}

Desenvolveu-se estudo de coorte com gestantes adolescentes atendidas nos serviços de atenção pré-natal e acompanhamento nutricional de uma maternidade pública da cidade do Rio de Janeiro. Foram investigadas aquelas que apresentavam gestação de feto único e eram livres de enfermidades crônicas.

As gestantes foram captadas no momento do registro na unidade para o pré-natal e somente foram investigadas aquelas que tiveram o Termo de Consentimento Livre e Esclarecido assinado, no caso das menores de 18 anos, por um responsável, ou pela própria gestante, quando maior de idade. O acompanhamento ocorreu desde o primeiro trimestre de gestação até o pós-parto imediato. Foram incluídas, no presente estudo, as gestantes que receberam pelo menos duas consultas de acompanhamento nutricional durante o pré-natal: a primeira até a $13^{\mathrm{a}}$ semana gestacional (ou no primeiro trimestre) e a segunda entre a $14^{\mathrm{a}}$ e a $28^{\mathrm{a}}$ semana (ou no segundo trimestre de gestação).

Os dados foram coletados a partir de informações dos prontuários e de questionários estruturados em entrevistas pessoais durante as sessões de aconselhamento nutricional no primeiro e no segundo trimestres de gestação. Foram obtidas informações sobre o consumo de alimentos, dados sociodemográficos e história obstétrica. As gestantes foram examinadas quanto aos perfis antropométrico, clínico, funcional e bioquímico.

Foram coletadas informações referentes à idade materna, cor da pele (por autoclassificação), situação marital (casada/solteira), escolaridade (ensino fundamental completo ou incompleto), condições de saneamento da moradia (adequadas/inadequadas), número de consultas da assistência pré-natal, número de consultas do acompanhamento nutricional durante o pré-natal e intercorrências durante a gestação (diabetes gestacional, síndrome hipertensiva gestacional, infecção urinária).

A tomada das medidas antropométricas obedeceu às recomendações do Ministério da Saúde ${ }^{7}$. Foram tomadas as medidas da estatura e do peso gestacional na primeira consulta de pré-natal e no pré-parto. O peso informado ou medido até a $13^{\mathrm{a}}$ semana gestacional foi considerado como peso pré-gestacional. Aferiu-se o peso das gestantes - que estavam descalças, usavam roupas leves e sem adereços -, por meio de uma balança mecânica da marca Filizola com capacidade para $120 \mathrm{~kg}$. A aferição da estatura foi realizada com régua antropométrica de 2 metros e graduada em milímetros, acoplada à mesma balança ${ }^{7}$.

Para avaliação do estado nutricional, classificou-se o Índice de Massa Corporal (IMC) pré-gestacional conforme os pontos de corte para meninas adolescentes estabelecidos pela Organização Mundial da Saúde ${ }^{8}$. A definição das faixas de ganho de peso gestacional semanal e total foi realizada segundo a recomendação do Ministério da Saúde ${ }^{7}$. Para gestantes que iniciam a gestação com baixo peso, é recomendado um ganho de 
peso total de $12,5 \mathrm{~kg}$ a $18,0 \mathrm{~kg}$; para aquelas com peso pré-gestacional adequado, um ganho total de $11,5 \mathrm{~kg}$ a $16,0 \mathrm{~kg}$; para as com sobrepeso pré-gestacional, de $7,0 \mathrm{~kg}$ a $11,5 \mathrm{~kg}$, e para as com obesidade pré-gestacional, um ganho de peso gestacional total de $7,0 \mathrm{~kg}$ a $9,1 \mathrm{~kg}^{7}$.

A ingestão dietética habitual foi estimada a partir de Questionário de Frequência de Consumo de Alimentos Semiquantitativo (QFCAS), aplicado em dois momentos: no primeiro e no segundo trimestre gestacional. O QFCAS foi elaborado incluindo os alimentos mais citados por gestantes adultas e adolescentes atendidas na unidade estudada. Era composto por 20 itens alimentares e comportava a inclusão de outros alimentos referidos pelas adolescentes. Foi solicitado às gestantes que relatassem as quantidades e a frequência com que cada item foi consumido no período anterior à última consulta (consumo diário, semanal, quinzenal ou mensal); ou, ainda, indicar se não consumiu o alimento nenhuma vez no período de referência (nunca).

A quantidade em medida de massa ou volume (gramas ou mililitros) das porções de referência dos itens listados no QFCAS foi estimada com base em tabela de medidas caseiras ${ }^{9}$. Após a conversão de frequência de consumo relatada em frequência diária, a quantidade usualmente consumida por dia para cada item alimentar foi estimada multiplicando-se quantidade relatada pela frequência diária de consumo.

O consumo de energia, de macro e de micronutrientes foi estimado com base nas tabelas de composição de alimentos do United States Department of Agruculture (USDA, Departamento de Agricultura dos Estados Unidos) ${ }^{10}$, do Instituto de Nutrición Centro America y Panamá $(\text { INCAP) })^{11}$ e da Tabela Brasileira de Composição de Alimentos (TACO) ${ }^{12}$.

As informações sobre os recém-nascidos foram coletadas dos prontuários: idade gestacional, peso e comprimento ao nascer. O peso ao nascer foi classificado em inadequado $(<2500 \mathrm{~g})$ e adequado ( $\geq 2500 \mathrm{~g})$. A idade gestacional ao nascer foi estimada pela Data da Última Menstruação
(DUM) e foi classificada em pré-termo (idade gestacional $<37$ semanas), a termo (entre 37 e 42 semanas) e pós-termo ( $\geq 42$ semanas) ${ }^{7}$.

Aplicou-se o teste de Kolgomorov-Smirnov para avaliar a simetria das distribuições das variáveis contínuas e foram calculadas as medidas de tendência central e de dispersão para cada período de observação. O consumo de nutrientes foi descrito para os dados ajustados pelo consumo de energia total segundo o método de resíduos ${ }^{13}$.

Testou-se a associação entre variáveis categóricas e o peso ao nascer classificado em adequado e inadequado pelo teste do Qui-quadrado $\left(\chi^{2}\right)$. Foram aplicados os testes $t$ de Student (para amostras independentes) e Mann-Whitney para testar a igualdade das médias de ingestão de energia e nutrientes (ajustadas pelo consumo de energia) segundo as categorias de adequação do peso ao nascer e da idade gestacional ao nascer, e a igualdade das variâncias foi avaliada pelo teste de Levene.

Foram desenvolvidos modelos de regressão linear múltipla para identificar os fatores associados ao peso ao nascer, nos quais foram incluídas as variáveis associadas ao peso ao nascer e a idade gestacional nas análises univariadas com valor de $p<0,20$. A significância estatística considerada no modelo final foi de $5 \%$.

O estudo foi planejado respeitando-se os aspectos éticos previstos pelo Conselho Nacional de Saúde ${ }^{14}$ e aprovado pelo Comitê de Ética da Maternidade-Escola da Universidade Federal do Rio de Janeiro ( $n^{\circ}$ 1758.0.000.361-07, de 29/06/2007).

\section{RES U L T A D O S}

Entre 2007 e 2010, foram acompanhadas 227 gestantes adolescentes a partir do primeiro trimestre de gestação até o puerpério imediato. Dessa amostra, foram selecionadas 139 (61,2\%) que tinham recebido pelo menos duas consultas com o nutricionista durante o pré-natal: uma no primeiro trimestre e outra no segundo trimestre de gestação. 
A idade das gestantes variou de 13,0 a 19,7 anos, com média de 17,2 anos (Desvio-Padrão-DP $=1,6$ anos); $62,2 \%$ eram solteiras ou viviam sem o companheiro; $95,5 \%$ residiam em condições adequadas de saneamento; 65,4\% concluíram o ensino fundamental e 70,3\% eram não brancas. A paridade média foi de $1,2(\mathrm{DP}=0,4)$ e o intervalo intergestacional médio foi de 23,3 meses ( $D P=13,9$ meses).

O ganho de peso gestacional médio apresentado foi de $14,1 \mathrm{~kg}$ ( $D P=5,3 \mathrm{~kg})$. A média do número de consultas de assistência pré-natal foi de 7,0 ( $D P=2,2$ consultas) e do número de sessões de orientação nutricional no pré-natal foi de 4,6 ( $D P=1,1$ sessões). As intercorrências gestacionais mais prevalentes foram anemia $(33,5 \%)$, amniorrexe prematura $(2,6 \%)$, síndrome hipertensiva gestacional $(1,9 \%)$ e infecção do trato urinário $(1,9 \%)$. Observou-se que $74,1 \%$ das gestantes iniciaram a gestação com o peso adequado e $35,3 \%$ apresentaram ganho de peso gestacional total adequado.

A média de peso ao nascer foi de $3200 \mathrm{~g}$ ( $D P=400 \mathrm{~g}$ ) e a idade gestacional média ao nascer

Tabela 1. Características sociodemográficas e antropométricas de gestantes adolescentes atendidas em uma Maternidade Pública do Rio de Janeiro, entre 2007 e 2010, de acordo com o peso ao nascer de seus conceptos.

\begin{tabular}{|c|c|c|c|c|c|}
\hline Características & $\begin{array}{c}\text { PN adequado } \\
(\geq 2,5 \mathrm{~kg})(n=134)\end{array}$ & $\%$ & $\begin{array}{l}\text { PN inadequado } \\
(<2,5 \mathrm{~kg})(\mathrm{n}=5)\end{array}$ & $\%$ & $\begin{array}{c}\text { Significância } \\
\text { estatística* }\end{array}$ \\
\hline Idade materna & & & & & 0,53 \\
\hline Até 15 anos & 10 & 100,0 & 0 & 0,0 & \\
\hline$>15$ anos & 124 & 96,1 & 5 & 3,9 & \\
\hline Cor & & & & & 0,66 \\
\hline Branca & 39 & 97,5 & 1 & 2,5 & \\
\hline Não branca & 95 & 96,0 & 4 & 4,0 & \\
\hline Saneamento & & & & & 0,50 \\
\hline Adequado & 123 & 96,1 & 5 & 3,9 & \\
\hline Inadequado & 11 & 100,0 & 0 & 0,0 & \\
\hline Estado civil & & & & & 0,34 \\
\hline Solteira & 82 & 97,6 & 2 & 2,4 & \\
\hline Casada/vive com companheiro & 52 & 94,5 & 3 & 5,5 & \\
\hline Instrução & & & & & 0,80 \\
\hline Ensino fundamental completo & 88 & 96,7 & 3 & 3,3 & \\
\hline Ensino fundamental incompleto & 45 & 95,7 & 2 & 4,3 & \\
\hline Número de consultas da assistência pré-natal & & & & & 0,96 \\
\hline Até 6 consultas & 51 & 96,2 & 2 & 3,8 & \\
\hline$\geq 6$ consultas & 83 & 96,5 & 3 & 3,5 & \\
\hline Número de sessões de acompanhamento nutricional & & & & & 0,90 \\
\hline Até 4 sessões & 14 & 87,5 & 2 & 12,5 & \\
\hline$>4$ sessões & 120 & 97,5 & 3 & 2,5 & \\
\hline Adequação do ganho de peso gestacional total & & & & & 0,77 \\
\hline Adequado & 48 & 94,1 & 3 & 5,9 & \\
\hline Inadequado & 86 & 97,7 & 2 & 2,3 & \\
\hline Estado nutricional pré-gestacional & & & & & 0,58 \\
\hline Baixo peso & 5 & 100,0 & 0 & 0,0 & \\
\hline Adequado & 93 & 94,9 & 5 & 5,1 & \\
\hline Sobrepeso & 28 & 100,0 & 0 & 0,0 & \\
\hline Obesidade & 8 & 100,0 & 0 & 0,0 & \\
\hline
\end{tabular}

Nota: *Teste Qui-quadrado; PN: Peso ao Nascer. 
foi de 38,9 semanas ( $D P=1,7$ semanas); $96,4 \%$ dos recém-nascidos apresentaram peso adequado ao nascer ( $\geq 2500 \mathrm{~g}$ ) e 5,3\% dos recém-nascidos foram classificados como pré-termo ( $<37$ semanas de gestação).

Não foi observada associação entre as características sociodemográficas, as variáveis assistência pré-natal e antropométricas maternas e a adequação do peso ao nascer (Tabela 1). A ingestão materna de energia e de nutrientes não variou conforme as características sociodemográficas das gestantes, assim como o ganho de peso gestacional total e idade gestacional ao parto (dados não mostrados).
Não foram observadas diferenças significativas nas médias de ingestão de energia e nutrientes (ajustadas pelo consumo energético) segundo as categorias de adequação do peso ao nascer para o primeiro trimestre de gestação (Tabela 2).

No segundo trimestre gestacional, verificou-se que não houve diferenças nas médias de consumo de energia segundo as categorias de adequação do peso ao nascer; porém, gestantes com bebês de peso ao nascer adequado apresentaram consumo médio mais elevado de proteínas $(p=0,02)$, lipídeos $(p=0,02)$, ácidos graxos

Tabela 2. Ingestão de energia, de macro e de micronutrientes no primeiro trimestre gestacional, segundo a adequação do peso ao nascer de conceptos das gestantes adolescentes atendidas em Maternidade Pública do Rio de Janeiro entre os anos de 2007 e 2010.

\begin{tabular}{|c|c|c|c|c|c|c|c|}
\hline \multirow{2}{*}{ Nutrientes } & \multicolumn{3}{|c|}{$P N$ adequado $(\geq 2,5 \mathrm{~kg})(n=134)$} & \multicolumn{3}{|c|}{ PN adequado $(<2,5 \mathrm{~kg}) \quad(n=5)$} & \multirow{2}{*}{$\begin{array}{l}\text { Significância } \\
\text { estatística* }\end{array}$} \\
\hline & Mediana & Média & (IC95\%) & Mediana & Média & $(\mathrm{IC} 95 \%)$ & \\
\hline Energia (kcal) & 2922,0 & 3053,0 & $(2849-3299)$ & 2648,0 & 2630,0 & $(1222-4038)$ & 0,49 \\
\hline \multicolumn{8}{|l|}{ Macronutrientes ${ }^{1}$} \\
\hline Proteínas (g) & 84,0 & 86,0 & $(81-90)$ & 87,0 & 85,3 & $(67-108)$ & 0,89 \\
\hline Carboidratos (g) & 410,0 & 410,0 & $(399-424)$ & 426,0 & 436,0 & $(394-478)$ & 0,29 \\
\hline Lipídeos (g) & 72,0 & 77,0 & $(71-81)$ & 65,0 & 63,0 & $(54-71)$ & 0,16 \\
\hline Ácidos graxos saturados (g) & 26,6 & 29,0 & $(26,7-31,2)$ & 23,5 & 24,3 & $(21,1-27,5)$ & 0,28 \\
\hline Ácidos graxos poli-insaturados (g) & 17,4 & 20,1 & $(17,7-22,0)$ & 16,3 & 15,7 & $(4,8-26,7)$ & 0,49 \\
\hline Ácidos graxos monoinsaturados(g) & 21,0 & 21,6 & $(19,9-23,0)$ & 17,5 & 17,0 & $(14,8-19,3)$ & 0,12 \\
\hline Ácidos graxos ômega-3 (g) & 2,6 & 2,8 & $(2,5-2,9)$ & 2,6 & 2,6 & $(1,2-3,9)$ & 0,83 \\
\hline Colesterol (mg) & 303,0 & 323,0 & $(290-352)$ & 268,0 & 2450,0 & $(170-329)$ & 0,36 \\
\hline \multicolumn{8}{|l|}{ Vitaminas $^{1}$} \\
\hline Vitamina $\mathrm{A}(\mu \mathrm{g})$ & 854,0 & 1238,0 & $(1031-1458)$ & 819,0 & 783,0 & $(316-1250)$ & 0,71 \\
\hline Vitamina C (g) & 72,0 & 128,0 & $(99-158)$ & 96,0 & 114,0 & $(-82-310)$ & 0,98 \\
\hline Folato (g) & 529,0 & 512,0 & $(477-548)$ & 541,0 & 512,0 & $(293-731)$ & 0,92 \\
\hline Tiamina $(g)$ & 2,3 & 3,4 & $(1,6-5,4)$ & 2,5 & 9,6 & $(-13,9-33,2)$ & 0,37 \\
\hline Riboflavina (g) & 2,6 & 2,7 & $(2,5-2,8)$ & 2,5 & 2,7 & $(2,2-3,2)$ & 0,87 \\
\hline Niacina (g) & 25,6 & 26,0 & $(24,2-28,1)$ & 22,3 & 21,9 & $(17,3-26,6)$ & 0,44 \\
\hline \multicolumn{8}{|l|}{ Minerais $^{1}$} \\
\hline Cálcio (g) & 642,0 & 667,0 & $(606-730)$ & 617,0 & 655,0 & $(392-917)$ & 0,96 \\
\hline Ferro $(\mathrm{g})$ & 11,0 & 12,0 & $(11-12)$ & 12,0 & 12,0 & $(11-12)$ & 0,69 \\
\hline Magnésio (g) & 282,0 & 295,0 & $(276-316)$ & 305,0 & 299,0 & $(244-355)$ & 0,56 \\
\hline Zinco (g) & 12,6 & 13,0 & $(12,3-13,8)$ & 12,2 & 12,4 & $(8,7-16,1)$ & 0,82 \\
\hline Cobre (g) & 2,2 & 3,1 & $(2,9-3,7)$ & 2,2 & 2,1 & $(1,2-3,0)$ & 0,76 \\
\hline Potássio (g) & 2762,0 & 2867,0 & $(2706-3057)$ & 2766,0 & 2705,0 & $(1808-3601)$ & 0,86 \\
\hline Sódio (g) & 1412,0 & 1468,0 & $(1298,4-1626,5)$ & 1331,0 & 1444,0 & $(498-2389)$ & 0,98 \\
\hline Fósforo (g) & 1345,0 & 1314,0 & $(1254-1375)$ & 1321,0 & 1299,0 & $(1122-1478)$ & 0,78 \\
\hline Manganês (g) & 6,2 & 7,1 & $(6,6-7,7)$ & 6,2 & 6,1 & $(5,3-7,0)$ & 0,76 \\
\hline
\end{tabular}

Nota: ${ }^{*}$ Teste Mann-Whitney; ${ }^{1}$ Ajustado pelo consumo de energia pelo método de resíduos ${ }^{13}$. IC95\%: Intervalo de Confiança $95 \%$; PN: Peso ao Nascer. 
saturados $(p=0,02)$ e monoinsaturados $(p=0,05)$, colesterol $(p=0,01)$, cálcio $(p \leq 0,01)$, potássio $(p=0,01)$ e fósforo $(p \leq 0,01)$. Entretanto, o consumo de carboidratos foi maior naquelas que tiveram bebês com peso ao nascer inadequado $(p=0,02)$. Não foram observadas diferenças significativas entre as médias de consumo de vitaminas segundo a adequação do peso ao nascer (Tabela 3).

Observou-se, em modelo de regressão multilinear, que a idade gestacional ao parto, o número de consultas da assistência pré-natal e o consumo de ácidos graxos poli-insaturados, de ácidos graxos ômega-3 e de colesterol no segundo trimestre da gestação associaram-se ao incremento do peso ao nascer independentemente do número de sessões da assistência nutricional pré-natal, do ganho de peso gestacional total, da paridade, do número de abortos e da ingestão de energia, carboidratos, proteínas, vitaminas e minerais no segundo trimestre da gestação (Tabela 4).

\section{DISCUSS ÃO}

Observou-se que o consumo de proteínas, lipídeos, cálcio, fósforo e potássio associou-se ao peso ao nascer adequado, independentemente

Tabela 3. Ingestão de energia, de macro e de micronutrientes no segundo trimestre, segundo a adequação do peso ao nascer de conceptos das gestantes adolescentes atendidas em uma Maternidade Pública do Rio de Janeiro entre 2007 e 2010.

\begin{tabular}{|c|c|c|c|c|c|c|c|}
\hline Nutrientes & \multicolumn{3}{|c|}{ PN adequado $(\geq 2,5 \mathrm{~kg})(\mathrm{n}=134)$} & \multicolumn{3}{|c|}{ PN adequado $(<2,5 \mathrm{~kg})(\mathrm{n}=5)$} & $\begin{array}{l}\text { Significância } \\
\text { estatística* }\end{array}$ \\
\hline \multicolumn{8}{|l|}{ Macronutrientes $^{1}$} \\
\hline Proteínas (g) & 87 & 87 & $(84-92)$ & 73 & 62 & $(27-97)$ & 0,02 \\
\hline Ácidos graxos saturados (g) & 25,1 & 26,2 & $(24,6-28,1)$ & 18,2 & 15,6 & $(3,1-28,0)$ & 0,02 \\
\hline Ácidos graxos poli-insaturados (g) & 13,9 & 15,4 & $(13,6-17,0)$ & 4,6 & 6,2 & $(-5,3-17,8)$ & 0,05 \\
\hline Ácidos graxos monoinsaturados(g) & 18,2 & 19,3 & $(18,1-20,5)$ & 11,7 & 11,7 & $(-1,4-23,5)$ & 0,05 \\
\hline Ácidos graxos ômega-3 (g) & 2,9 & 2,9 & $(2,8-3,1)$ & 2,1 & 2,1 & $(1,0-3,2)$ & 0,07 \\
\hline Vitamina C (g) & 49 & 110 & $(82-143)$ & 20 & 45 & $(-80-170)$ & 0,37 \\
\hline Folato $(\mathrm{g})$ & 495 & 521 & $(488-543)$ & 585 & 544 & $(398-690)$ & 0,56 \\
\hline Tiamina (g) & 2,4 & 2,4 & $(2,3-2,5)$ & 2,6 & 2,7 & $(1,9-3,4)$ & 0,28 \\
\hline Riboflavina (g) & 1,9 & 1,9 & $(1,7-2,0)$ & 1,7 & 1,3 & $(1,7-2,6)$ & 0,27 \\
\hline Niacina (g) & 24,4 & 26,2 & $(24,6-27,8)$ & 25,4 & 26,4 & $(13,7-39,2)$ & 0,71 \\
\hline \multicolumn{8}{|l|}{ Minerais $^{1}$} \\
\hline Cálcio (g) & 815 & 765 & $(700-876)$ & 330 & 176 & $(-442-794)$ & $\leq 0,01$ \\
\hline Ferro (g) & 11,3 & 11,7 & $(11,2-12,7)$ & 11,1 & 10,8 & $(6,7-15,0)$ & 0,67 \\
\hline Magnésio (g) & 308 & 308 & $(294-322)$ & 289 & 249 & $(150-348)$ & 0,14 \\
\hline
\end{tabular}

Nota: *Teste Mann-Whitney e $t$ student; ${ }^{1}$ Ajustado pelo consumo de energia pelo método de resíduos ${ }^{13}$. IC95\%: Intervalo de Confiança $95 \%$; PN: Peso ao Nascer. 
558 | ABF CAMPOS et al.

Tabela 4. Variáveis preditoras ${ }^{1}$ do peso ao nascer (em gramas) de conceptos de gestantes adolescentes atendidas em uma Maternidade Pública do Rio de Janeiro entre 2007 e 2010.

\begin{tabular}{|c|c|c|}
\hline Variáveis & $\begin{array}{l}\text { Desfecho: peso ao nascer } \\
\text { (gramas) } \beta(\text { IC } 95 \%)\end{array}$ & $\begin{array}{c}\text { Significância } \\
\text { estatística }\end{array}$ \\
\hline Idade gestacional ao parto (em semanas) & $\begin{array}{c}105,79 \\
(46,11-120,40)\end{array}$ & $<0,01$ \\
\hline Número de consultas de pré-natal & $\begin{array}{c}34,14 \\
(2,04-58,05)\end{array}$ & 0,04 \\
\hline Ingestão de lipídeos no segundo trimestre da gestação (gramas) & $\begin{array}{c}3,06 \\
(1,52-3,84)\end{array}$ & 0,05 \\
\hline Ingestão de ácidos graxos ômega-3 no segundo trimestre da gestação (gramas) & $\begin{array}{c}74,33 \\
(10,73-123,14)\end{array}$ & 0,04 \\
\hline Ingestão de ácidos graxos poli-insaturados no segundo trimestre da gestação (gramas) & $\begin{array}{c}7,49 \\
(0,62-12,63)\end{array}$ & 0,04 \\
\hline Ingestão de colesterol no segundo trimestre da gestação (miligramas) & $\begin{array}{c}0,38 \\
(0,01-0,64)\end{array}$ & 0,04 \\
\hline
\end{tabular}

Nota: ${ }^{1}$ Modelo de regressão linear múltipla tendo como variável dependente o peso ao nascer (em gramas) e ajustado por: número de sessões da assistência nutricional pré-natal, ganho de peso gestacional total, paridade, número de abortos, ingestão de energia, de carboidratos e de proteínas no segundo trimestre da gestação. IC95\%: Intervalo de Confiança de 95\%.

do número de consultas de pré-natal e da idade gestacional no parto, o que evidencia a importância da dieta durante a gravidez em adolescentes.

As gestantes que tiveram seus conceptos com adequado peso ao nascer apresentaram consumo mais elevado de proteínas, cujas principais fontes foram carne vermelha e branca, leite e derivados e feijão. A ingestão adequada de proteínas durante a gestação tem importância para o desenvolvimento da placenta, para a expansão dos tecidos maternos, inclusive para o aumento no volume sanguíneo e para o crescimento do feto $^{15}$.

Sloan et al..$^{16}$ avaliaram a associação do consumo de proteína durante a gravidez com o peso ao nascer de recém-nascidos em mulheres adultas e adolescentes, e verificaram que aquelas que relataram consumo mais elevado de proteínas tiveram filhos com o peso ao nascer significativamente maior quando comparadas com as gestantes com consumo de proteína abaixo desse limite.

O consumo de lipídeos totais, de ácidos graxos poli-insaturados, de ácidos graxos ômega-3 e de colesterol influenciou o peso ao nascer de conceptos de gestantes adolescentes. No grupo estudado, as principais fontes de ácidos graxos poli-insaturados e ômega-3 foram os óleos vegetais utilizados no preparo dos alimentos e o feijão. Todos os ácidos graxos acumulados pelo feto provêm da circulação materna através da transferência placentária, e essa dependência é demonstrada por estudos que indicam forte correlação positiva materno-fetal para todos os ácidos graxos essenciais ${ }^{17}$.

As características dos lipídeos que compõem a dieta materna influenciam o perfil dos ácidos graxos presentes nas membranas celulares, na formação de estruturas uteroplacentárias, no desenvolvimento do sistema nervoso central e na acuidade visual do feto ${ }^{18}$. Alguns estudos têm contribuído para levantar a hipótese de que o aumento do consumo de ácido graxo ômega-3 está relacionado ao prolongamento da gestação, devido à redução na produção de prostaglandinas e incremento na produção de prostaciclinas, levando ao relaxamento do miométrio e prorrogando o início do trabalho de parto ${ }^{19}$.

Estudo de meta-análise realizado por Szajewska et al. ${ }^{20}$ demonstrou que a utilização 
de suplementação com ácido graxo ômega-3 durante a gestação prolongou a duração da gestação em 1,6 a 2,6 dias, o que é considerado um fator de proteção para o concepto, na medida em que propicia maior tempo para o ganho de peso intrauterino e a diminuição da morbidade e mortalidade relacionadas com a prematuridade. Outros estudos também observaram associação significativa entre o consumo de lipídeos e o peso ao nascer adequado. Raol et al. ${ }^{21}$, ao avaliarem a dieta de 797 gestantes adolescentes e adultas, observaram que um maior consumo de lipídeos na segunda semana gestacional se associou com adequado peso ao nascer de seus conceptos. Olsen et al. ${ }^{22}$ avaliaram a ingestão de ômega-3 em 99 dinamarquesas grávidas e verificaram que a alta ingestão desse ácido graxo associou-se ao aumento médio na duração da gravidez de 5,7 dias, o que contribuiu para uma maior adequação do peso ao nascimento em comparação com aquelas com consumo inferior a esse limite.

As gestantes adolescentes, que ainda estão em fase de desenvolvimento ósseo, têm necessidades aumentadas de minerais que promovem a mineralização óssea, como cálcio, fósforo e potássio. A deficiência no consumo desses nutrientes pode estar associada ao prejuízo no crescimento e desenvolvimento fetal, como foi demonstrado em ensaio clínico desenvolvido por Villar \& Repke ${ }^{23}$ com 189 gestantes adolescentes nos Estados Unidos. Os resultados revelaram que a suplementação de cálcio durante a gestação resultou em recém-nascidos com peso ao nascer maior em comparação aos filhos das adolescentes que não receberam a suplementação. Bang \& Sun Lee $^{24}$ avaliaram a dieta de 234 gestantes adultas residentes na Coreia, e o consumo de fósforo foi associado significativamente com a adequação do peso ao nascer.

O consumo dietético das adolescentes grávidas analisadas nesse estudo foi comparável ao observado em estudo nacional de base populacional que evidenciou prevalência importante de inadequação no consumo de vitaminas lipossolúveis, vitamina $C$, magnésio, e principalmente cálcio e fósforo entre adolescentes brasileiros avaliados na Pesquisa de Orçamentos Familiares 2008-2009 25 .

A idade gestacional ao parto e o número de consultas de pré-natal foram variáveis preditoras do peso ao nascer. Assinale-se que a idade gestacional ao parto também é influenciada pelo consumo alimentar, e a assistência pré-natal apropriada tem papel importante na duração da gestação. O efeito protetor do pré-natal no resultado obstétrico de adolescentes foi descrito em estudo realizado com 3118 puérperas adolescentes residentes no município do Rio de Janeiro por Granado et al. ${ }^{26}$, que mostrou que a assistência pré-natal foi eficiente na prevenção da prematuridade e do baixo peso ao nascer. Resultados semelhantes foram relatados por Kilsztajn et al. ${ }^{27}$, que observaram o aumento do número de consultas pré-natais como fator redutor do baixo peso ao nascer e de parto pré-termo tanto em gestantes adultas como em adolescentes.

A dificuldade na aferição do consumo alimentar pode ser considerada uma possível limitação do estudo, visto que não se dispõe de métodos capazes de determinar o consumo alimentar com exatidão ${ }^{28}$. Porém, o questionário de frequência de consumo de alimentos ainda é considerado adequado para avaliar o consumo habitual, e, por isso, é amplamente utilizado em estudos epidemiológicos ${ }^{28}$.

A prevalência do baixo peso ao nascer observada neste estudo foi reduzida em relação ao observado em outros estudos com gestantes adolescentes ${ }^{29,30}$ : possivelmente esse achado expresse as características da população de estudo, que recebeu atenção pré-natal diferenciada, com atendimento nutricional.

Com base nos resultados observados, é possível indicar que esforços devem ser envidados no sentido de tornar o acompanhamento nutricional uma atividade de rotina nos serviços de atenção pré-natal. Essa prática poderia contribuir para a minimização da ocorrência de resultados perinatais adversos, particularmente o baixo peso ao nascer, especialmente em gestantes adolescentes, que constituem grupo com alta vulnerabilidade nutricional. 


\section{O N CLUS Ã O}

Este estudo permitiu evidenciar que o consumo alimentar da gestante adolescente tem impacto sobre a situação nutricional do concepto e que o perfil lipídico da dieta da mãe foi o principal preditor do peso ao nascer.

Em modelos multiajustados, foram observadas associações significativas entre as exposições analisadas (consumo de energia e nutrientes) e o desfecho investigado (baixo peso ao nascer), apesar de sua reduzida prevalência no grupo investigado. Dessa forma, os achados deste estudo reiteram a necessidade e a importância da orientação nutricional durante o pré-natal e da atenção que deve ser dedicada à alimentação de adolescentes grávidas.

\section{COLABORADORES}

ABF CAMPOS colaborou na coleta de dados, análise do banco de dados, tratamento e análise estatística dos dados; concepção e revisão do manuscrito; RA PEREIRA colaborou na supervisão do tratamento e análise dos dados, concepção, revisão e edição final do manuscrito; co-orientadora da dissertação de mestrado que deu origem ao manuscrito. J QUEIROZ colaborou na coleta de dados, análise estatística dos dados e revisão do manuscrito final. C SAUNDERS colaborou na concepção do estudo, supervisão da coleta de dados, armazenamento e análise do banco de dados, tratamento e análise estatística dos dados; concepção, revisão e edição final do manuscrito; orientadora da dissertação de mestrado que deu origem ao manuscrito.

\section{REFERÊ N CIAS}

1. Chen XK, Wen SW, Fleming N, Demissie K, Rhoads GG, Walker M. Teenage pregnancy and adverse birth outcomes: A large population based retrospective cohort study. Int J Epidemiol. 2007; 36(2):368-73.

2. American Dietetic Association. Position of the American Dietetic Association: Nutrition and lifestyle for a healthy pregnancy outcome. J Am Diet Assoc. 2008; 108(3):553-61.
3. Barros DC, Pereira RS, Gama SGN, Leal MC. O consumo alimentar de gestantes adolescentes no município do Rio de Janeiro. Cad Saúde Pública. 2004; 20(Supl 1):S121-S9.

4. Anon. The time to prevent disease begins before conception: DOHaD position paper. World Nutr. 2011; 2(4):195-205.

5. Bertin RL, Parisenti J, Di Pietro PR, Vasconcelos FAG. Métodos de avaliação de consumo alimentar de gestantes: uma revisão. Rev Bras Saúde Matern Infant. 2006; 4(6):383-90.

6. Lima GSP, Sampaio HAC. Influência de fatores obstétricos, socioeconômicos e nutricionais da gestante sobre o peso do recém-nascido: estudo realizado em uma maternidade em Teresina, Piauí. Rev Bras Saúde Mater Infant. 2004; 4(3):253-61.

7. Brasil. Ministério da Saúde. Pré-natal e puerpério: atenção humanizada e qualificada: manual técnico. Brasília: MS; 2006.

8. World Health Organization. Protein and amino acid requirements in human nutrition: Report of a joint WHO/FAO/UNU expert consultation. Geneva: WHO; 2007. WHO Technical Report Series, 935.

9. Pinheiro $A B V$, Lacerda EMA, Benzecry EH. Tabela para avaliação de consumo alimentar em medidas caseiras. 5a ed. São Paulo: Atheneu; 2005.

10. Gebhardt SE, Cutrufelli RL, Howe JC, Pehrsson P, Lemar L, Haytowitz D, et al. USDA National Nutrient Database for Standard Reference, release 19; 2006 [cited 2010 Jan 17]. Available from: <http://www. ars.usda.gov/research/publications/publications. htm?SEQ_NO_115=199178>.

11. Instituto de Nutrición Centro America y Panamá Tabla de composición de alimentos para uso en America Latina. Guatemala: Incamp; 2006 [acceso 2010 enero 13]. Disponible en: <http://www.incap. org.gt/>.

12. Universidade Estadual de Campinas. Núcleo de Estudos e Pesquisas em Alimentação. Tabela brasileira de composição de alimentos - TACO. Versão 2. $2^{\text {a }}$ ed. Campinas: Unicamp; 2006.

13. Willet WC, Howe GR, Kushi LH. Adjustament for total energy intake in epidemiologic studies. Am J Clin Nutr. 1997; 65(4 Suppl):1220S-8S.

14. Brasil. Ministério da Saúde. Diretrizes e normas regulamentadoras de pesquisas envolvendo seres humanos. Resolução n 196/96 do Conselho Nacional de Saúde. Rio de Janeiro: Fundação Oswaldo Cruz; 1998.

15. Hamaoui E, Hamaoui M. Nutritional assessment and support during pregnancy. Gastroenterol Clin North Am. 2003; 32(1):59-121. 
16. Sloan NL, Lederman SA, Leighton J, Himes JH, Rush $D$. The effect of prenatal dietary protein intake on birth weight. Nutr Res. 2001; 21(1-2):129-39.

17. Fleith M, Clandinin MT. Dietary PUFA for preterm and term infants: Review of clinical studies. Crit Rev Food SciNutri. 2005; 45(3):205-29.

18. Silva DRB, Miranda Junior PF. A importância dos ácidos graxos poliinsaturados de cadeia longa na gestação e lactação. Rev Bras Saúde Matern Infant. 2007; 7(2):123-33.

19. Jordan RG. Prenatal omega-3 fatty acids: Review and recommendation. J Midwifery Womens Health. 2010; 55(6):520-8.

20. Szajewska H, Horvath A, Koletzko B. Effect of n-3 long-chain polyunsaturated fatty acid supplementation of women with low-risk pregnancies on pregnancy outcomes and growth measures at birth: A meta-analysis of randomized controlled trials. Am J Clin Nutr. 2006; 83(6):1337-44.

21. Rao S, Yajnik CS, Kanade A, Fall CHD, Margetts $B M$, Jackson AA, et al. Intake of micronutrient-rich foods in rural Indian mothers is associated with the size of their babies at birth: Pune maternal nutrition study. J Nutr. 2001; 131(4):1217-24.

22. Olsen SF, Hansen HS, Sommer S, Jensen B, Sorensen $\mathrm{TI}$, Secher NJ, et al. Gestational age in relation to marine $n-3$ fatty acids in maternal erythrocytes: A study of women in the Faroe Islands and Denmark. Am J Obstet Gynecol. 1991; 164(5):1203-9.

23. Villar J, Repke JT. Calcium supplementation during pregnancy may reduce preterm delivery in hight risk population. Am J Obstet Gynecol. 1990; 163(4): 1124-31.
24. Bang SW, Sun Lee S. The factors affecting pregnancy outcomes in the second trimester pregnant women. Nutr Res Pract. 2009; 3(2):134-40.

25. Instituto Brasileiro de Geografia e Estatística. Pesquisa de orçamentos familiares 2008-2009: análise da disponibilidade domiciliar de alimentos no Brasil: 2011. Rio de Janeiro: IBGE; 2011 [acesso 2011 out 6]. Disponível em: <http://www.ibge. gov.br/home/ estatistica/populacao/condicaodevida/pof/2008_ 2009_analise_consumo/pofanalise_2008_2009. $\mathrm{pdf}>$.

26. Granado SNG, Szwarcwald CL, Leal M. Experiência de gravidez na adolescência, fatores associados e resultados perinatais entre puérperas de baixa renda. Cad Saúde Pública. 2002; 18(1):153-61.

27 Kilsztajn S, Rossabach AC, Carmo MSN, Sugahara GTL. Assistência pré-natal, baixo peso e prematuridade no Estado de São Paulo, 2000. Rev Saúde Pública. 2003; 37(3):303-11.

28. Holanda LB, Barros Filho, AA. Métodos aplicados em inquéritos alimentares. Rev Paul Pediatr. 2006; 24(1):62-70.

29. Pardo RA, Nazer J, Cifuentes L. Prevalencia al nacimiento de malformaciones congénitas y de menor peso de nacimiento en hijos de madres adolescentes. Rev Med Chile. 2003; 131(10):1165-72.

30. Simões VMF, Silva AAM, Bettiol H, Lamy-Filho F, Tonial SR, Mochel EG. Características da gravidez na adolescência em São Luís, Maranhão. Rev Saúde Pública. 2003; 37(5):559-65.
Recebido em: 28/8/2012 Versão final em: 9/4/2013 Aprovado em: 2/5/2013 
